\title{
Fourteen-Month-Olds Adapt Their Imitative Behavior in Light of a Model's Constraints
}

\author{
Kata Gellén ${ }^{1}$ and David Buttelmann ${ }^{1,2}$ \\ 1 "Kleinkindforschung in Thüringen", University of Erfurt, Erfurt, Germany \\ ${ }^{2}$ Department of Developmental Psychology, University of Bern, Bern, Switzerland
}

Correspondence should be addressed to Kata Gellén; kata.gellen@uni-erfurt.de

Received 19 April 2016; Revised 2 September 2016; Accepted 17 November 2016; Published 9 January 2017

Academic Editor: Magdalena Janus

Copyright (C) 2017 K. Gellén and D. Buttelmann. This is an open access article distributed under the Creative Commons Attribution License, which permits unrestricted use, distribution, and reproduction in any medium, provided the original work is properly cited.

\begin{abstract}
Rather than reenacting every action they observe, preverbal infants adapt their imitative behavior. Although previous studies have revealed the capability of preverbal infants to imitate selectively, the question about the adaptability of this behavior on an individual level did not attract considerable scientific attention until now. In the current study, we investigated whether 14-month-old infants flexibly alternate their imitative response in accordance with a model's changing physical constraints in a body-part imitation paradigm. Participants were presented with two novel actions whereby a model illuminated a light-box and turned on a soundbox, either by using her forehead (head touch) or by sitting on the apparatus (sit-touch). Each participant observed these tasks in two conditions: once where the model's hands were occupied and once where her hands were free while executing the head or sit-touch. Participants were more likely to reenact the observed novel behavior when the model had freely chosen to perform it than when she had to do so due to physical constraints. Not only did we replicate a number of previous findings, we show here that preverbal infants adapt their imitative behavior across conditions based on the physical constraints of the model. These results point towards the adaptable nature of imitative behavior also on an individual level. This ability might be one of the building blocks for children for learning their social group's specific action repertoire.
\end{abstract}

\section{Introduction}

In order to conform to their social community, humans must master a wide range of social behaviors. An intense learning process of these behaviors dominates the first years of an infant's life. When it comes to the acquisition of opaque, arbitrary forms of human culture [1], imitation of others' behavior is considered a central means of learning. It has been found that 12- to 18-month-old infants learn one to two novel actions every day, merely by observing others around them [2]. Rather than imitating everything and everyone, however, infants tend to be selective in their imitative behavior, a tendency that is present from around 12 months of age $[3,4]$. Several factors influence infants' rates of imitation, some of which concern the action itself (for action component saliency see [5]), but also the context in which the action is presented (for pedagogical versus nonpedagogical context see [6]; for the role of emotional feedback on an action see [7]), as well as the model's previously displayed competence [8] and her group membership [9].

The adaptable nature of imitation becomes evident when infants face the choice of reenacting a novel action that they observe under different circumstances. In order to investigate whether preverbal infants would base their action choice on efficiency evaluation, Gergely et al. [10] presented a novel head action (task adapted from [11]) to two groups of 14-month-olds whereby the model illuminated a lightbox with her forehead. While one group of infants observed the unusual head action with the model freely resting her hands next to the lamp (hands-free condition), the other group observed a demonstration of the identical head action with the single modification that the model had her handsoccupied by holding a blanket wrapped around her torso (hands-occupied condition). Participants were never constrained in the response phase which took place with a one-week delay after the demonstration. The majority (69\%) 
of infants in the hands-free condition reenacted this novel means, whereas only $21 \%$ of infants did so in the handsoccupied condition. According to the teleological account [12], infants consider others as rational agents who aim to achieve a goal by the most efficient available means. Consequently, the authors proposed that different imitation rates in the Gergely et al. [10] study were due to children's evaluation of the model's action plan as being rational in the light of her available means. While the model did not have any available means to illuminate the light-box other than her head in the hands-occupied condition, in the hands-free condition she could have decided to use her hands instead of her head. Infants are highly familiar with the efficacy of hand actions as they explore and manipulate objects with their hands from an early age [13]. When they witnessed the head action in the hands-free condition, infants are taken to assume that this action was chosen by the model due to it being a more efficacious means than the familiar option of hand use. The tendency for infants to be ambitious to learn effective actions made them copy what they assumed the most efficacious means in the Gergely et al. [10] study; that is the use of the hand in the hands-occupied condition, and the use of the head in the hands-free condition. This leads to the argument that selective imitation is rational in nature.

To see whether infants engage in rational imitative behavior in object-on-object manipulations or if it is a behavior specific to body-part imitation, researchers developed other paradigms that entail tool use and the demonstration of an action with puppets. In the majority of these studies, conditions with alternating physical constraints were introduced to infants in between-subjects designs (e.g., [3]). The only exception, to the best of our knowledge, is the study of Buttelmann et al. [14], which involved a tool-use task presented in a within-subjects design. Here, 14-montholds observed the target actions in two conditions that placed differing observable constraints on an actor. In the response phase infants got the chance to interact with the apparatuses and reach a goal by either using the demonstrated (unusual) tool or to opt for using only their hands (Studies 1 and 2) or a more usual tool (Study 3). Like Gergely and colleagues [10], these aforementioned studies found that preverbal infants were more likely to imitate the model's object manipulation when the model had freely chosen to perform the unusual action, in comparison to when it was the only available means to achieve the goal due to physical constraints. The question, however, remains as to whether the adaptable nature of infants' imitative responses, which was revealed by Buttelmann and colleagues [14], is a characteristic that encompasses tasks and characterizes true imitative behavior. Since Buttelmann et al.s [14] study utilized a tool-use paradigm, due to the nature of the task, infants' responses might be perceived as object movement reenactment, whereby it is only the physical course of the object (i.e., the tool) that is attended, rather than infants' reasoning about the choice of action plan itself in relation to the intended goal [15]. In contrast, body-part imitation does not face the same criticism and is considered as true imitation [16].

While a wide range of paradigms has been applied with a between-subjects design, clearly there is a serious lack of varying methodologies for studies within the field that apply a within-subjects design. The application of a bodypart imitation paradigm in a within-subjects design provides a deeper insight into the extent of selective imitative behavior on an individual level. It can potentially reveal whether infants are able to flexibly adapt their body movements in a similar manner to their tool usage based on their evaluation of others' actions in various alternating conditions. It appears that humans' closest genetic relatives, chimpanzees, are capable of adapting their imitative response in accordance with a human demonstrator's changing physical constraints: enculturated chimpanzees imitated the use of a specific body part rationally when given the chance to do so immediately after they observed a human demonstrator operating novel apparatuses [17]. In each of three sessions, the chimpanzees observed two of the three applied unusual actions (head, sit, or foot action); one in the hands-free and one in the handsoccupied condition. In order to decrease carry-over effects, half of the apparatus illuminated upon operation while the other half produced a sound. Similarly to human infants in Gergely et al.s [10] between-subjects design, enculturated chimpanzees imitated the unusual action to a higher degree in the hands-free (37.5\% of trials) than in the hands-occupied condition (18.8\% of trials) in this within-subjects design. The current study adapted two tasks (head-touch and sit-touch task) and the within-subjects design of Buttelmann et al.s [17] in order to reveal whether the same flexibility exists also in human infants on an intrapersonal level.

As for the underlying mechanisms for adaptable imitative behavior, besides the teleological account [12] three other major theories have been proposed to explain why infants differentially imitate in the light of a model's alternating physical constraints. Buttelmann et al. [14] argued that infants consider the head- or the sit-touch action as the model's execution of her intention, that is, her rational choice of action plan. In the hands-occupied condition, infants infer the reason for why the model used an unusual means: because her hands were physically constrained she had to use her head. In the hands-free condition, by contrast, the model's rationale for choosing the unusual means is not obvious, which makes infants imitate the novel action, possibly to determine why the model formed this intention. Thus, what makes infants imitate novel actions according to this account is the lack of apparent reasons for the model's choice of means. Rather than attributing mental-state understanding to infants and considering selective imitation as being based on a rational decision, Paulus et al. [18] argue in their two-stage model of infant imitation that, first, it is the observed action effect that is decisive in whether or not an observed action will be later imitated. Second, they argue that infants' ability to map the observed action onto their own motor repertoire shapes their imitative behavior. They claim that higher imitation rates in the hands-free condition are due to infants' capability to execute the head action when they support themselves with their hands on the table, similarly to how the model demonstrated the action in this condition. By contrast, 14-month-olds are unable to map the head action when the model did not use her hands for support, as that is how the model executed the head 
action in the hands-occupied condition, and are therefore less likely to imitate in this condition (see [19], for an integrative model that combines the teleological account and the twostage model of infant imitation). Similarly, the perceptual distraction approach [20] also explains the phenomenon of rational imitation by considering the more basic processes at work, suggesting at it being selective rather than rational. According to this approach, infants in the hands-occupied condition are distracted by the unusual look of the blanket tightly wrapped around the model's torso. Therefore, they are less able to focus on the modeled head action itself, resulting in lower imitation in comparison to the hands-free condition, wherein no comparable attentional interference is present. Importantly, the four accounts have identical predictions of infants' overall imitation tendency in the classical one-trial hands-free and hands-occupied conditions.

In the current study, we have, however, administered two trials in each condition, instead of presenting infants with each task only once. This could potentially influence imitation rates in the hands-free and the hands-occupied conditions differently according to the different theoretical accounts. Following the principles of the teleological account [12] or the mentalistic account [14], it is unlikely that a repeated presentation of the unusual action in the hands-occupied condition has any effect on infants' imitation rates: Since the model is still physically constrained, she still cannot use her hands. In contrast, in the hands-free condition, the two accounts might differ in their predictions. Since infants in the response phase of the first trial learned that the hands are a very effective means of operating the apparatus (see [10], for the finding that virtually all participants used their hands when given the chance to operate the apparatus), when observing the model demonstrating the novel action despite her hands being free a second time, infants might know that this cannot be the most efficacious means. They might thus be less likely to imitate in the second trial according to the teleological account. Regarding the mentalistic account [14], the model's use of her head in the second trial of the hands-free condition might emphasize her intentionality, which might lead to an increase of imitation of the unusual modeled action in the second trial. An increase in imitation rate would be also expected by the perceptual distraction account [20]. However, this increase should take place in the hands-occupied condition, whereby the repeated exposure to the demonstration decreases the level of distraction by the blanket obscuring the hands in the second trial. No differences in imitation level should occur in the hands-free condition. In contrast, the two-stage model of infant imitation [18] would predict similar imitation rates in both trials in both conditions, as the action effect stays constant across demonstrations and no change takes place between trials which would improve infants' ability to map the observed action onto their own motor repertoire in the second trial.

Infants' ability to flexibly adapt their behavior to changing contexts has been widely studied in paradigms other than imitation, such as in the A-not-B task. These studies established a continuous increase in the ability to switch with a significant leap improvement at around one year of age (see, e.g., [21]), which is the age at which infants start to engage in selective imitation, when tested in a between-subjects design [4]. Therefore, the question arises as to whether a similar flexibility is present in their imitative behavior. The purpose of the current study was to investigate infants' reactive flexibility in terms of their ability to adapt their imitative responses to changing situational constraints of the model by presenting the same infants with both the handsfree and the hands-occupied conditions within one testing session. Previously, in studies that investigated infants' ability to adjust their behavior to changing contextual information, the situational constraints that made infants display a flexible behavior directly affected the infants. For example, 18-montholds relied selectively on the social cues of their caregivers while descending on a challenging slope, depending on whether they were wearing none, normal, or Teflon-soled shoes to alter their locomotive abilities [22]. Infants used the social information provided by their caregivers in challenging situations, when they were uncertain of their motor abilities but did not do so when they felt secure; that is, they adapted their social information use behavior in accordance with their own physical circumstances. In contrast, in the current study the constraints were applied only to the model and thereby could only have an indirect influence on infants' behavior.

As currently only one study has been published that investigated whether selective imitative behavior of human infants is present in a within-subjects design [14], one has to be cautious in drawing far-reaching conclusions about the extent of the behavior's apparently adaptable nature on an individual level. Therefore, the current study aims at examining whether infants can effectively adapt their own behavior not only from one task to another (i.e., the head-touch and the sit-touch task) but whether they do so with regard to others' alternating behavior in the light of their observable constraints. This is important in order to identify the extent or possible limits of infants' learning abilities. The application of a body-part imitation paradigm ensures that infants' response behavior can be considered as true imitation and provides the basis for a direct comparison to classical studies of selective imitation. In the case that rational imitative behavior is present in an adaptable form on an intrapersonal level at the age of 14 months, infants would adapt their behavior in accordance with the model's changing physical constraints when they are presented with both the hands-free and the hands-occupied condition. Infants would overall display higher imitation in the hands-free condition in comparison to the hands-occupied condition. For the investigation of which theoretical account (i.e., the teleological account, the mentalistic account, the two-stage model of infant imitation, or the perceptual distraction approach) might explain our data best, we will analyze the first and second trials separately and match our findings to the predictions outlined above.

\section{Method}

2.1. Participants. Eighty-two 14-month-olds participated in the current study ( $\mathrm{M}=13$ months; 30 days; range = 13 months; 2 days-14 months; 26 days; 48 girls). An additional 3 infants were tested but had to be excluded from the analysis as they had no valid trials due to inattentiveness $(n=1)$ or due 
to a combination of experimenter and apparatus error $(n=$ 2 ). Infants were recruited from a database of parents who volunteered to participate in child developmental studies at a mid-sized German university.

2.2. Materials. For both the head-touch and the sit-touch tasks, two apparatuses were used, one of which elicited a melody and one that lit up when pressed on the top (a total of four apparatuses) (Figure 1). The four apparatuses applied in the two tasks were a head-touch sound apparatus $(28 \mathrm{~cm}$ long $\times 25.8 \mathrm{~cm}$ wide $\times 19.6 \mathrm{~cm}$ high; tilted at $50.5^{\circ}$ ), a headtouch light apparatus $(22.6 \mathrm{~cm}$ long $\times 25.8 \mathrm{~cm}$ wide $\times 20 \mathrm{~cm}$ high; tilted at $32.5^{\circ}$; the lamp mounted on the box was $11.5 \mathrm{~cm}$ in diameter), a sit-touch sound apparatus $(15.5 \mathrm{~cm}$ high $\times$ $32.5 \mathrm{~cm}$ in diameter), and a sit-touch light apparatus $(60.1 \mathrm{~cm}$ long $\times 22 \mathrm{~cm}$ wide $\times 20.9 \mathrm{~cm}$ high). The experimenter $(\mathrm{E}$, always female, $n=2$ ) performed the head action on the apparatuses while sitting at the table's side adjacent to the infant (as the head-touch apparatuses were tilted at angle, the original setup whereby E sits opposite to the participant could not be applied). She demonstrated the sit-touch action in a similar sideway position to ensure a better view of the demonstration.

2.3. Design and Procedure. Infants participated in two imitation tasks wherein they observed the novel target actions of the head touch and sit-touch (adapted from [17]). In a single session, each task was presented in two successive trials, for a total of four trials. Each trial entailed a demonstration phase followed by an immediate response phase. A withinsubjects design was applied: infants observed one of the novel actions executed in the hands-free condition and the other in the hands-occupied condition. One action was executed on a sound- and the other on a light apparatus. The order of the tasks, the presented modalities, and the conditions were counterbalanced across participants. Children were randomly assigned to one of eight possible groups, which were created by counterbalancing the binary variables of condition, task, and modality of apparatus (Table 1).

After a ten-minute warm-up session, wherein the assistant (A, always female, $n=2$ ) explained the procedure of the study to the parent, A took her seat in the testing room and waited for $\mathrm{E}$ to accompany participants into the testing room. Throughout the study E played the role of the model, and A's task was to arrange the setup and place the apparatuses in front of $\mathrm{E}$ and the infants. The inclusion of two differing tasks was necessary, as infants at the age of 14 months have a strong tendency to generalize their experiences in imitation tasks from one situation to another [23]. In order to enable comparison of the performance of infants in the current study and that of enculturated chimpanzees, the current procedure followed the procedure applied by Buttelmann et al. [17] as closely as possible.

Before the first task, E used a small ball to play with the infants for approximately 1-2 minutes in order to familiarize them with the study room before starting the testing process. After the completion of the first task (i.e., two trials), E presented infants with a toy bear and played with them shortly to provide time for A to change the room setting for the second task (i.e., two trials).

Head-Touch Task. During the head-touch task, infants sat on their parent's lap adjacent to a table. While E turned away from the table to remove the toy that she had used before the task, A brought the head-touch apparatus and placed it on the table in front of E's seat ("So, this comes here"). After turning back towards the table, E acknowledged the apparatus ("Oh, great!") and took her seat. She then shivered as if she were cold and asked A for a blanket. What happened next differed between conditions. In the hands-free condition, $\mathrm{E}$ wrapped the dark blue blanket around her torso, then freed her hands with the blanket still resting on her shoulders, lifted her hands and slightly wiggled her fingers close to the apparatus, signaling clearly to the infant that she could freely move them, and placed them next to the apparatus onto the table's surface. After calling for the attention of the infant ("Participant's name, pay attention!"), E executed the novel head-touch action. She turned on the apparatus to elicit sound or light by pressing its top with her forehead, with the pressing action lasting approximately 3 seconds. E performed the demonstration three times. In case the child did not pay attention to the actions, $\mathrm{A}$ signaled to $\mathrm{E}$ and she repeated the demonstration to ensure that the infant observed three of them. This was necessary to ensure that any differences in infants' imitative behavior were not due to their differing experience level with the novel action. Additional repetition of the demonstration was necessary for eight infants in eight trials. After the demonstration phase, E turned her head towards the infant, in front of whom A placed the apparatus, and encouraged the child to play with it ("And now you!").

The hands-occupied condition differed only in the actions of the model with the blanket. After receiving the blanket from A, E wrapped it around her body but kept her hands underneath, holding the blanket from below. She kept her hands in the same position, obscured under the blanket, until the end of the second trial.

Sit-Touch Task. The sit-touch task took place on the floor. After having played with the ball or the toy bear while the infant sat on a small carpet on the floor, E instructed the parent to take their child onto their lap and took the ball away. While E walked to the side of the room with her back to the infant in order to get a box, A placed the sit-touch apparatus approximately 1.5 meters away from the infants' carpet ("So, this comes here") and returned to her seat at the side of the room. Upon her return, E acknowledged the apparatus ("Oh, great!"). The procedure from this point onwards differed between conditions. In the hands-free condition, E placed the empty cardboard box, which she was holding in her hands when returning to the centre of the room, next to her feet. She then called for the child's attention ("Participant's name, pay attention!") and then demonstrated the novel sit-touch action by squatting over and sitting on top of the apparatus three times. Each sitting action lasted approximately 3 seconds. Since all infants paid close attention, no additional repetition of the demonstration for this task was necessary for any of the infants. After the demonstration phase, E picked up 


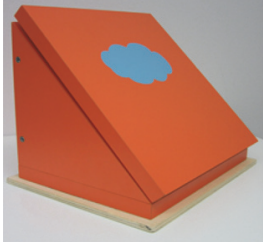

(a)

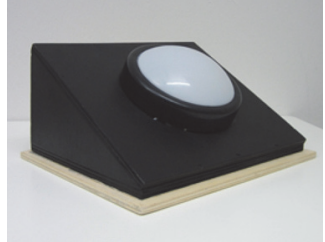

(b)

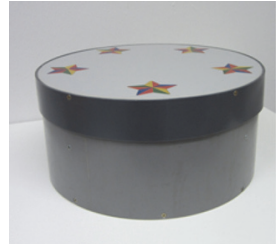

(c)

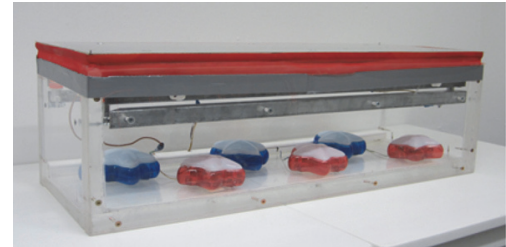

(d)

FIGURE 1: The apparatuses used in the study: (a) head-touch sound apparatus; (b) head-touch light apparatus; (c) sit-touch sound apparatus; (d) sit-touch light apparatus.

TABLE 1: Overview of the counterbalanced orders of tasks, conditions, and modalities.

\begin{tabular}{lcc}
\hline Overall order number & 1st task $(2$ consecutive trials $)$ & 2nd task (2 consecutive trials) \\
\hline 1 & Head-touch, light, hands-free & Sit-touch, sound, hands-occupied \\
2 & Head-touch, light, hands-occupied & Sit-touch, sound, hands-free \\
3 & Sit-touch, sound, hands-free & Head-touch, light, hands-occupied \\
4 & Sit-touch, sound, hands-occupied & Head-touch, light, hands-free \\
5 & Sit-touch, light, hands-free & Head-touch, sound, hands-occupied \\
6 & Sit-touch, light, hands-occupied & Head-touch, sound, hands-free \\
7 & Head-touch, sound, hands-free & Sit-touch, light, hands-occupied \\
8 & Head-touch, sound, hands-occupied & Sit-touch, light, hands-free \\
\hline
\end{tabular}

the box from the floor, took a seat on a chair opposite the infant, placed the empty cardboard box next to herself, and encouraged the child to engage with the apparatus ("Participant's name, now you!"). Parents had been previously instructed to let their child down onto the carpet upon this signal.

In the hands-occupied condition, E followed the same procedure as in the hands-free condition, with the exception that she kept the empty cardboard box in her hand while carrying out the target sit-touch action, as well as when being seated during the response periods of the task.

The response phase followed a standardized procedure across all conditions, tasks, and modalities: an initial waiting period of a maximum of 60 seconds within which time inactive infants were encouraged by $\mathrm{E}$ to interact with the apparatus at 10-second intervals ("Now you!") was introduced to control for personality traits, for example, shyness, which were not in the interest of the current study. Following the time of infants' first touch of the apparatuses, the following durations were applied for the two imitation tasks: 60 seconds for the head-touch and 120 seconds for the sit-touch task. This deviation from the procedure of Buttelmann et al. [17] in the current study was introduced because of the pilot findings of Zmyj et al. [8] concerning human infants' differing interest in the apparatuses and the differing difficulty levels that the tasks posed for them. In both tasks, from E's signal, the infant had 60 seconds to touch the top of the apparatus. If no touch took place within this time period, the trial was over, and A walked back to the table, took the apparatus ("And now it is E's turn again"), and placed it back in front of E. If the infant touched the apparatus within the 60 seconds after E's signal, a 60 -second/120 second response phase started from the time of the first touch on the top of the apparatus, and A removed the apparatus only after the entire response period elapsed. The demonstration phase and the response period were then repeated once more, following the identical procedure.

2.4. Coding and Analysis. The first author coded infants' actions from recorded videotapes. The behavior of infants with at least one valid trial (out of the four administered trials), in which children interacted with the apparatus or were inactive but had the entire 60 seconds waiting period available to touch the apparatus, was included in the analysis, which resulted in 272 of 328 possible data points (i.e., $82.93 \%$ of all possible data points; 48 infants had 4 valid trials; 15 infants had 3 valid trials; 16 infants had 2 valid trials; and 3 infants had 1 valid trial; in 192 trials were infants inactive: 18 infants had 4 valid trials; 22 infants had 3 valid trials; 23 infants had 2 valid trials; and 8 infants had 1 valid trial). Infants who only had one valid trial or two valid trials within the same condition were not excluded from the analysis as their behavior can potentially contribute valuable insights to imitation behavior on an interpersonal level and were therefore included in the between-subjects analyses. The remaining 56 trials were excluded from the analysis due to infants being fussy $(n=25)$, experimenter error $(n=14)$, apparatus failure $(n=12)$, or parental error $(n=5)$.

The focus of interest was whether or not children reenacted the target actions. Firstly, in order to compare human infants' performance to that of enculturated chimpanzees [17] and the evidence presented by Gergely et al. [10] the first 20 seconds of the response phase was analyzed. In addition, infants' behavior across the entire response period (i.e., headtouch task: 60 seconds; sit-touch task: 120 seconds; similarly to $[8,24])$ was also of interest to determine whether results 
remained constant or infants displayed another behavioral pattern beyond the classically investigated 20 -second duration. An action was coded as a head-touch if infants touched the lamp with any part of their head. According to a similar principle, sit-touch was coded when children sat on the sit-touch apparatus or attempted to do so by kneeling on its top (see $[8,24])$. For each valid trial infants received a score that signaled whether the target action was imitated (1) or not (0) to allow analysis of the individual trials. These scores were then transformed into percentages, according to task (head-touch/sit-touch), condition (hands-free/handsoccupied), and modality (sound/light) and for the separate blocks of trials, indicating for what proportion of the trials infants replicated the observed actions (i.e., each infant received on each block either $0 \%$ for not imitating the target action, 50\% for imitating in one trial, or $100 \%$ for imitating in both trials of a task). A naïve coder, blind to the purpose of the study, coded $25 \%$ of the videos for the presence of the target action in order to ensure the reliability of the coding. The interrater agreement was excellent for both the head-touch and the sit-touch task (20 seconds and entire response phase: Cohen's kappa of 1.0). Furthermore, the interrater agreement was also excellent for the body part used to first touch the apparatus (Cohen's kappa of 1.0).

\section{Results}

3.1. Preliminary Analyses. No main effect of task order or order of condition or of gender was present, either for the 20 -seconds period or for the entire response period. Furthermore, no differences between tasks were revealed, either for the 20-seconds response period or for the entire response period. When examining the differences between modalities, analysis revealed similar imitation behavior in the case of the sound and the light apparatuses within the 20-second response period and within the entire response period.

Additional analyses revealed that the only influence on participants' performance we found was that of order of modalities at a trend level within the first 20 seconds (MannWhitney $=472.50, N_{\text {light-sound }}=40, N_{\text {sound-light }}=30$, $p=.057, r=.23)$ and at a significant level across the full response period (Mann-Whitney $=435.50, N_{\text {light-sound }}=40$, $N_{\text {sound-light }}=30, p=.019, r=.28$ ) (for description see Table 2). Infants who started with the task involving the sound modality did not display evidence of adaptable imitative behavior between conditions. By contrast, those who started with the light apparatus and received the sound modality in the second block of trials imitated the target actions significantly more often in the hands-free than in the hands-occupied condition (20 seconds: Wilcoxon test, $Z=$ $-2.81, N=40, p=.005, r=.44$; entire response period: Wilcoxon test, $Z=-2.60, N=40, p=.009, r=.41$; for description see Table 2).

To examine whether imitation rates, in the separate conditions, differed across trials within the single blocks, we compared imitation rates in the first and the second trials of each separate blocks. In the case that the first administered block entailed the hands-free condition, imitation rates were higher in the second trial than in the first trial of the same block (20 seconds: $\chi^{2}(1,29)=5.784, p=.033$; entire response period: $\chi^{2}(1,29)=17.532, p<.0001$, for description see Table 3 ). In the case that the first administered block utilized the hands-occupied condition, results yielded only a trend within the first 20 seconds $\left(\chi^{2}(1,33)=15.984, p=\right.$ $.061)$. The analysis of the entire response period, however, revealed a significantly higher imitation rate in the second trial in comparison to the first trial $\left(\chi^{2}(1,33)=21.290, p=\right.$ $.006)$. The comparison of imitation rates in the hands-free condition administered in the second block showed higher imitation rates in the second trial than in the first (20 seconds: $\chi^{2}(1,27)=12.420, p=.017$; entire response period: $\chi^{2}$ $(1,27)=14.850, p=.003)$. The comparison of trials in the hands-occupied condition administered in the second block showed no difference in imitation rates between trials within the first 20 seconds $\left(\chi^{2}(1,31)=5.373, p=.161\right)$ but revealed higher imitation rates in the second trial within the duration of the entire response phase $\left(\chi^{2}(1,31)=7.330\right.$, $p=.045)$.

In order to rule out the idea that any difference in infants' imitation rates between conditions might be the result of infants' differing success rate of turning on the apparatuses by hand, we compared infants' successful hand actions in their active trials in the hands-free and the hands-occupied conditions. We found that infants were able to successfully turn on the apparatus at least once by using their hand in the hands-free condition in $68.97 \%$ of the trials $(\mathrm{SD}=43.76)$ and in the hands-occupied condition in $63.93 \%$ of the trials $(\mathrm{SD}=46.67)$, which did not result in a difference between conditions (Wilcoxon test, $Z=-.929, N=48, p=.353$ ). This means that a possible difference in infants' imitation between conditions is unlikely to be the result of a difference in infants' unsuccessful hand use between conditions.

3.2. Main Analyses. The novel focus of our study was to investigate whether selective imitative behavior was also present on an individual level in human infants. Upon analyzing the first 20 seconds of the response period, infants' rate of imitation differed significantly between conditions, with a higher imitation rate in the hands-free than in the hands-occupied condition (Wilcoxon test, $Z=-2.327, N=$ $70, p=.02, r=.28$, Figure 2 ). The results were similar across the entire response period, as the unusual action was imitated at a higher rate in the hands-free condition than in the handsoccupied condition (Wilcoxon test, $Z=-2.288, N=70$, $p=.022, r=.27$; Figure 2). Infants used first their hands in $98.3 \%$ of the first touches in the case of the head-touch task and in $96.1 \%$ of the first touches in the case of the sit-touch task.

In order to check whether infants' performance differed without any experience gained within the current experiment, we analyzed the first administered block separately. Selective imitation was also present on a between-subjects level in infants' first block of trials. The imitation rate of the head- or sit-touch action was significantly higher in the hands-free than in the hands-occupied condition $(20 \mathrm{sec}-$ onds: Mann-Whitney $U=578.50, N_{\text {free }}=38, N_{\text {occupied }}=40$, $p=.005, r=.32$; entire response period: Mann-Whitney $\left.U=596.00, N_{\text {free }}=38, N_{\text {occupied }}=40, p=.014, r=.28\right)$ 
TABLE 2: Infants' mean imitation rate depending on the administered order of modalities.

\begin{tabular}{|c|c|c|c|c|c|c|c|}
\hline \multicolumn{4}{|c|}{ Light-sound } & \multicolumn{4}{|c|}{ Sound-light } \\
\hline \multicolumn{2}{|c|}{20 seconds } & \multicolumn{2}{|c|}{ Entire response period } & \multicolumn{2}{|c|}{20 seconds } & \multicolumn{2}{|c|}{ Entire response period } \\
\hline $\begin{array}{l}\text { Hands-free } \\
M(S D)\end{array}$ & $\begin{array}{c}\text { Hands-occupied } \\
\text { M (SD) }\end{array}$ & $\begin{array}{l}\text { Hands-free } \\
\text { M (SD) }\end{array}$ & $\begin{array}{c}\text { Hands-occupied } \\
\text { M (SD) }\end{array}$ & $\begin{array}{l}\text { Hands-free } \\
\text { M (SD) }\end{array}$ & $\begin{array}{l}\text { Hands-occupied } \\
\text { M (SD) }\end{array}$ & $\begin{array}{l}\text { Hands-free } \\
\text { M (SD) }\end{array}$ & $\begin{array}{l}\text { Hands-occupied } \\
\text { M (SD) } \\
\end{array}$ \\
\hline $22.5(37.47)$ & $5.0(18.95)$ & $30.0(43.56)$ & $8.8(27.47)$ & $13.3(31.98)$ & $11.7(28.42)$ & $15.0(35.11)$ & $15.0(29.8)$ \\
\hline
\end{tabular}

Note. The table includes the data of infants, in two groups depending on the order of administered modalities. The numbers represent percentages of trials. Values in parentheses represent standard deviations.

TABLE 3: Infants' mean imitation rates dependent of condition in the single trials of the separate administered blocks.

\begin{tabular}{lccccc}
\hline & $\begin{array}{c}\text { Analyzed section of } \\
\text { response period }\end{array}$ & $\begin{array}{c}\text { Hands-free condition } \\
\text { 1st administered trial }\end{array}$ & $\begin{array}{c}\text { 2nd administered trial } \\
\text { M (SD) }\end{array}$ & $\begin{array}{c}\text { 1st administered trial } \\
\text { M (SD) }\end{array}$ & $\begin{array}{c}\text { 2nd administered trial } \\
\text { M (SD) }\end{array}$ \\
\hline $\begin{array}{l}\text { 1st administered block } \\
\text { of trials }\end{array}$ & 20 seconds & $18.9(39.71)$ & $26.67(44.98)$ & $2.63(16.22)$ & $5.71(23.55)$ \\
2nd administered & Entire period & $21.62(41.73)$ & $33.33(47.94)$ & $5.26(22.63)$ & $8.57(28.40)$ \\
block of trials & 20 seconds & $10.81(31.48)$ & $13.79(35.09)$ & $6.06(24.23)$ & $15.15(36.41)$ \\
\hline
\end{tabular}

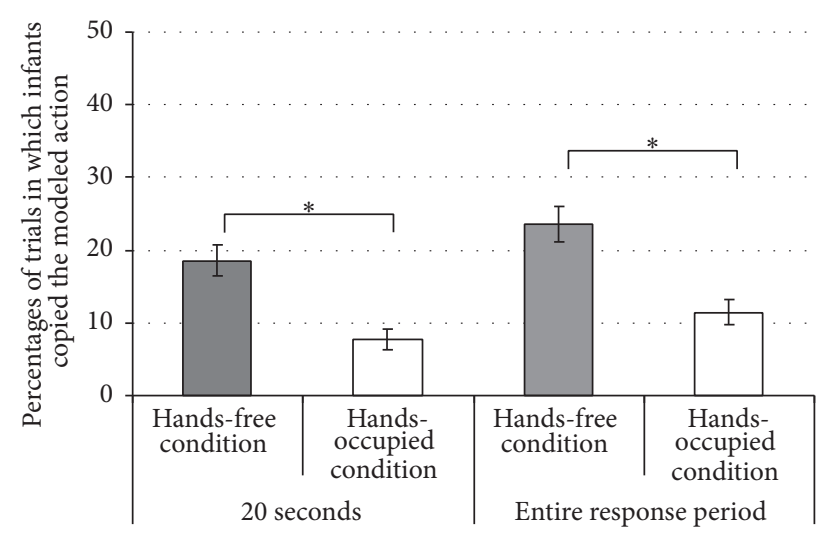

FIGURE 2: Infants' mean imitation rate within the first 20 seconds of and during the entire duration of the response period across tasks (within-subjects comparison). $*<.05$. Note: the mean percentages refer to the number of trials rather than to the number of participants; therefore no direct comparison can be made with the mean percentages reported by Gergely et al. [10], only with that of Buttelmann et al. [17].

(for description see Table 4). This difference in the first block of trials was already apparent in the first administered trial, as infants showed significantly alternating imitative behavior from the beginning onwards, with a higher rate of imitation in the hands-free in comparison to that of the hands-occupied condition (20 seconds: $\chi^{2}(1,75)=5.219, p=.022$; entire response period: $\chi^{2}(1,75)=4.341, p=.037$; for description see Table 4). This replicates the results of Gergely et al.'s [10] study within the first 20 seconds of the response period even in the case of applying two unusual target actions instead of only the classical head-touch task. The results remained similar for the second trial of the block, where infants also imitated the target actions also significantly more often in the hands-free than in the hands-occupied condition (20 seconds: $\chi^{2}(1,65)=5.448, p=.02$; entire response period: $\chi^{2}(1,65)=6.19, p=.013$; for description see Table 4$)$.

3.3. Comparison to Previous Studies. In addition, we wanted to assess whether we replicated the results of the previous studies upon which we based our design. In order to enable a direct comparison between the results of Gergely et al's [10] and the current study, firstly the imitative behavior of those infants who received the head-touch light apparatus as the first task was analyzed. These infants received the same task with the same modality as 14-month-olds in Gergely et al's [10] study with no prior experience of the other novel target action, that is, the sit-touch, within the framework of the study. Similarly to Gergely et al.s [10] study, infants in the current study imitated the head touch significantly more often in the hands-free than in the hands-occupied condition within the first 20 seconds of the response period even on the first administered trial (Chi-square test, $\chi^{2}(1,22)=7.062$, $p=.008$, for description see Table 4 ). The results remained the same for the entire response period (Chi-square test, $\chi^{2}(1,22)=7.062, p=.008$, for description see Table 4$)$. Furthermore, the imitation rates of this subgroup of infants were also higher in the hands-free condition than in the hands-occupied condition for the first administered block of trials (20 seconds: Mann-Whitney test, $U=28.0, N_{\text {free }}=9$, $N_{\text {occupied }}=14, p=.002, r=.64$; entire response period: Mann-Whitney test, $U=30.50, N_{\text {free }}=9, N_{\text {occupied }}=14$, $p=.008, r=.56$ ) (for description see Table 4 ). We thereby not only replicated the findings of Gergely et al. [10] but also extended those to a longer response period and to a block of two repeated trials.

As we adapted the experimental design from Buttelmann et al. [17], in the second step we analyzed the imitative behavior of those infants who received the light apparatuses in the first block, as enculturated chimpanzees always received the light apparatus before the sound apparatus in order to compare our results to those of the study with enculturated 
TABLE 4: Infants' mean imitation rates.

\begin{tabular}{|c|c|c|c|}
\hline \multirow[b]{2}{*}{ Analyzed response period } & \multirow{2}{*}{$\begin{array}{l}\text { Analyzed section of } \\
\text { response period }\end{array}$} & \multicolumn{2}{|c|}{ Condition } \\
\hline & & $\begin{array}{l}\text { Hands-free } \\
\text { M (SD) }\end{array}$ & $\begin{array}{c}\text { Hands-occupied } \\
\text { M (SD) }\end{array}$ \\
\hline \multirow{2}{*}{$\begin{array}{l}\text { 1st administered block of trials overall } \\
\text { (\% of trials, between-subjects) }\end{array}$} & 20 seconds & $21.05(36.08)$ & $3.75(17.5)$ \\
\hline & Entire period & $25.0(41.51)$ & $6.25(23.17)$ \\
\hline \multirow{2}{*}{$\begin{array}{l}\text { 1st administered trial } \\
\text { (\% of subjects, between-subjects) }\end{array}$} & 20 seconds & $18.92(39.71)$ & $2.63(16.22)$ \\
\hline & Entire period & $21.62(41.73)$ & $5.26(22.53)$ \\
\hline \multirow{2}{*}{$\begin{array}{l}\text { 2nd administered trial } \\
\text { (\% of subjects, between-subjects) }\end{array}$} & 20 seconds & $26.67(44.98)$ & $5.71(23.55)$ \\
\hline & Entire period & $33.33(47.95)$ & $8.57(28.4)$ \\
\hline \multirow{2}{*}{$\begin{array}{l}\text { 1st trial head-touch task, light condition, as the 1st task } \\
\text { (\% of subjects, between-subjects) }\end{array}$} & 20 seconds & $44.44(52.7)$ & $0.0(0.0)$ \\
\hline & Entire period & $44.44(52.7)$ & $0.0(0.0)$ \\
\hline \multirow{2}{*}{$\begin{array}{l}\text { Head-touch task (both trials), light condition, as the 1st task } \\
\text { (\% of trials, between-subjects) }\end{array}$} & 20 seconds & $44.44(46.4)$ & $0.0(0.0)$ \\
\hline & Entire period & $50.0(50.0)$ & $3.57(13.36)$ \\
\hline \multirow{2}{*}{$\begin{array}{l}\text { 1st administered block of trials, light condition } \\
\text { (\% of trials, within-subjects) }\end{array}$} & 20 seconds & $20.45(36.28)$ & $4.65(18.3)$ \\
\hline & Entire period & $27.27(42.39)$ & $8.14(26.57)$ \\
\hline
\end{tabular}

chimpanzees. Fourteen-month-olds, similarly to enculturated chimpanzees, reenacted the novel target action significantly more often in the hands-free condition than the handsoccupied condition within the first 20 seconds of the response period (Wilcoxon test, $Z=-2.810, N=40, p=.005$, $r=.44$, for description see Table 4). Furthermore, our results replicated those of Buttelmann et al. [17] when analyzing the entire response period (Wilcoxon test, $Z=-2.60, N=40$, $p=.009, r=.41$, for description see Table 4$)$.

\section{Discussion}

The main focus of the current study was to examine the adaptability of selective imitative behavior in 14-month-olds on an individual level. Our study yielded evidence that 14-montholds adapt their imitative behavior in accordance with the physical constraints of a model upon examining the first 20 seconds of the response period. Additionally, we found selective body-part imitation even for an extended duration of the entire response phase. Focusing on subgroups of participants, we replicated the findings of Gergely and colleagues [10] as well as Buttelmann and colleagues [17]. More specifically, our 14-month-old infants tailored their behavior in accordance with the model's physical constraints across conditions that were administered to them with a very short time delay. This short time delay demanded from infants the ability to immediately adapt their imitative responses in accordance with the model's changing constraints. Participants in the current study imitated the unusual target action significantly more often if the model demonstrated the action as her free choice than in the case when she was forced to do so because of some physical constraint. These findings suggest that preverbal infants, similarly to enculturated chimpanzees [17], have the capability of adapting their imitation of novel observed actions based on their perception of the constraints under which an action is modeled.

In the current study we replicated and extended a number of previous findings regarding selective imitation, for example, those of Gergely et al. [10]. That is, infants who received the head-touch light apparatus as the first administered task in the hands-free condition displayed a higher imitation rate within the first 20 seconds of their first trial than those infants who received the identical task and apparatus in the handsoccupied condition. This pattern remained constant even for the entire duration of the response period. We further extended these findings by additionally focusing on the adaptable nature of the skill on an individual level whereby we administered two target actions in two differing conditions to each participant. The results of our study show that selective imitation (irrespective of the underlying mechanism) is a true phenomenon, rather than a result of accidently allocating infants who are likely to imitate into the hands-free and the nonimitators into the hands-occupied condition. Infants predominantly imitated the target actions when they had observed them being carried out in the hands-free condition, wherein the use of the unusual body part was a freely chosen means that the model seemed to prefer over hand use. Imitation of the target actions was observed in spite of the predominant use of the most straightforward means, that is, the hands, as infants' first action (see also [10], for a similar finding). In contrast, the lower imitation rates in the handsoccupied condition suggest that when physical constraints were observable infants recognized that the model had to use a means other than the hands to operate the box (i.e., by her head or by sitting on it). As participants' hands were always free, they had the possibility of opting for using their hands to push the lid of the apparatus. These findings are also consistent with preverbal infants' selective tool-use tendency [14]. Importantly, as children engaged in similar behavior in both the head- and the sit-touch task, the extent of the adaptable nature of imitative skills is further highlighted across task types of body-part imitation paradigms.

Adapting the study design of Buttelmann et al. [17] enables the direct comparison of human infants' and enculturated chimpanzees' imitative behavior. Those infants, who started with the light modality in the current study (i.e., the same modality order as the one that enculturated chimpanzees received) showed a similar imitative response like did the chimpanzees in Buttelmann et al.s [17] study. Moreover, not only this subgroup's performance but also the 
performance of all infants in the current study showed a similar pattern to that of enculturated chimpanzees. In both cases, higher imitation of the demonstrated unusual action was observed in the hands-free condition in comparison to the hands-occupied condition, suggesting that both preverbal human infants and enculturated chimpanzees adapted their imitative behavior on the basis of the observed physical constraints of the model. In contrast, the behavioral pattern in the single trials shows a differing picture. While enculturated chimpanzees displayed higher imitation rates in their first trial than in their second trial in the hands-free condition, we found for infants a higher level of imitation in the second trial in this condition. Our results follow the same pattern as the findings of Schwier et al. [3], suggesting that while some enculturated chimpanzees may have learned across repeated trials that the use of the unusual body part was unnecessary, children's behavior was not steered by the same learning mechanism. Taking into account toddlers' and preschoolers' tendency to copy modeled actions irrespective of a model's circumstances or the necessity of all of the demonstrated single steps [25], it seems possible that 14-month-old infants not only imitate in order to learn how to operate a novel device but also do so in order to affiliate with the model [26]. Great apes, in contrast, seem to lack this social motivation and therefore stop imitating as soon as they figure out all possible ways to operate the novel device and then go for their own choice of means (e.g., $[24,27])$.

Four theoretical accounts provide an explanation to the behavioral pattern found by Gergely et al. [10] (i.e., the teleological account [12], the mentalistic account [14], the two-stage model of infant imitation [18], or the perceptual distraction account [20]). As stated earlier, the current study was not directly aimed at resolving the question which of these accounts is correct. In the hands-free condition, regardless whether it was administered in the first or in the second block, within both the first 20 seconds and within the duration of the entire response period, imitation rates were higher in the second than in the first trials. This would be in line with the principles of the mentalistic account [14], as the increase in the imitation rate might suggest infants' interpretation of the repeated demonstration of the unusual target action in the second trial as an emphasis on the intentional nature of the modeled action. This increase in imitation rates would, however, not be predicted by the teleological [12], the perceptual distraction account [20], or the two-stage model of infant imitation [18], as the model's rationality, motor action, or the appearance of the blanket did not change between trials. In the hands-occupied condition, the similar imitation rates in the first and second trial within the first 20 seconds provide support for the mentalistic [14] and the teleological account [12] and could also be explained by the two-stage model of infant imitation [18], as the model's physical constraints remained constant. It contradicts the prediction of the perceptual distraction account [20], as the position of the blanket remained the same across trials, and due to infants' habituation it should have presented a lower level of distraction in the second trial. This pattern, however, turned around when examining the entire duration of the response period. The increased imitation rate in the second trial is consistent solely with the prediction of the latter account. As the perceptual distraction account would suggest, infants might have habituated across the first trial to the unusual appearance of the blanket around the model's torso and been less distracted from the demonstrated head action in the second trial. Alternatively, a longer response period during which the model looks at the infant might result in a higher social pressure, whereby infants feel as they are expected to imitate the observed unusual action [26]. However, rather than solely relying on the comparison of single trials, developing conditions wherein the accounts would expect different rates of imitation (e.g., the button condition in [28], for a critical overview of the study see [29]) and presenting them to infants in future studies in a withinsubjects design could help disentangling the alternative explanations. These mixed results emphasize the necessity of expanding the pool of studies by applying not only various tasks and conditions but also a variety of response durations.

When analyzing our data for effects of the tasks, the modalities, or any of the orders in which those were applied, we found that infants predominantly imitated the observed novel action more often in the hands-free than the handsoccupied condition when they had the opportunity to interact first with a light apparatus. These findings might be explained by the differing demands that the various applied modalities placed on infants' attention. Based on findings that show whether or not an action results in an observable effect shapes the rate of imitation (e.g., $[30,31]$ ), it is likely that also the effect type influences imitative behavior. When presented with auditory-visual compounds infants prioritize the processing of auditory stimuli over that of visual stimuli $[32,33]$. This preference of processing auditory stimuli over visual stimuli when presented in parallel is due to attentional factors [34]. As the presence of an auditory stimulus is usually shorter than that of a visual one, it is reasonable to focus attention on what is audible rather than on what is visible. This makes sense especially early in the maturational process of attention abilities when attentional resources are limited. This resource allocation on the outcome of the modeled action, however, could potentially lead to a decreased level of attention paid to the visually observed action and its circumstances, which then might lead to a lower imitation rate.

An alternative explanation relies on a possible conflict of interest in the apparatus and in the unusual target action (see perceptual distraction account, [20]). In case of the light apparatus, the action effect was immediately visually connected to the demonstrated target action. Infants could directly observe how pushing the top of the light-boxes resulted in a light effect. In contrast, the location of the sound was not directly observable. It seems possible that the lack of observable location of the sound resulted in infants shifting their focus from imitating the target action to manually exploring the sound object (manual exploration and manipulation of objects is a behavior that is present already within the first year of life, see, e.g., [35]). Further research is necessary to establish how the differing action effect modalities and the demonstrated first action might influence infants' imitative behavior on the second task in spite of the fact that the utilized tasks and modalities were introduced 
to reduce possible carry-over effects. It is important to point out, however, that the study of Buttelmann et al. [17] did not include an order where the sound modality has been administered as the first modality of the task (i.e., in their study all subjects saw the light version of each task as the first modality). Therefore, it remains unclear how far the order of the administered modalities (presented in a randomized manner) influences great apes' imitative behavior. This order effect of modalities, thus, encourages further research to be conducted with human and nonhuman participants in the research of selective imitation across different modalities that include attentional input via only one or more channels.

Until now, to the best of our knowledge, no study has utilized the sit-touch task with infants younger than 14 months. The task would be, however, suitable for 12-month-olds (the youngest age group to date wherein selective imitative behavior has been observed [4]), as some children master the skill of walking around their first birthday [36]. Applying the identical tasks in order to reveal whether selective imitative behavior is present in an adaptable form even at 12 months of age or whether this aspect of the skill develops after infants' first birthday would shed more light into the ontogenetic development of the adaptability of selective imitation.

The importance of the present study lies in showing that selective imitative skills are adaptable when preverbal infants face novel behaviors demonstrated under opposing conditions. They can selectively imitate the use of unusual body parts not only across conditions, but also across tasks, which further emphasizes the adaptable nature of the skill even within a short timeframe. Being able to quickly adapt one's own behavior in the light of the circumstances that the demonstrator of an action faces enables infants to selectively imitate and learn only actions that are carried out intentionally. These actions are potentially important for the imitator to learn as they might be part of their social group's specific action repertoire.

\section{Competing Interests}

The authors declare that they have no competing interests.

\section{Acknowledgments}

The authors would like to thank all the caregivers and infants who participated in this study. Furthermore, they are thankful to Sarah Gruhne, Sarah Mercedes Mille, and Christina Predatsch for their assistance in data collection and to Jake Ryan Stanley for his useful comments on the manuscript.

\section{References}

[1] G. Gergely and G. Csibra, "Sylvia's recipe: the role of imitation and pedagogy in the transmission of cultural knowledge," in Roots of Human Sociality: Culture, Cognition, and Human Interaction, N. J. Enfield and S. C. Levenson, Eds., pp. 229-255, Berg Publishers, Oxford, UK, 2006.

[2] R. Barr and H. Hayne, "It's not what you know, it's who you know: older siblings facilitate imitation during infancy," International Journal of Early Years Education, vol. 11, no. 1, pp. 7-21, 2003.
[3] C. Schwier, C. van Maanen, M. Carpenter, and M. Tomasello, "Rational imitation in 12-month-old infants," Infancy, vol. 10, no. 3, pp. 303-311, 2006.

[4] N. Zmyj, M. M. Daum, and G. Aschersleben, “The development of rational imitation in 9- and 12-month-old infants," Infancy, vol. 14, no. 1, pp. 131-141, 2009.

[5] B. Elsner and C. Pfeifer, "Movement or goal: goal salience and verbal cues affect preschoolers' imitation of action components," Journal of Experimental Child Psychology, vol. 112, no. 3, pp. 283-295, 2012.

[6] I. Király, G. Csibra, and G. Gergely, "Beyond rational imitation: learning arbitrary means actions from communicative demonstrations," Journal of Experimental Child Psychology, vol. 116, no. 2, pp. 471-486, 2013.

[7] B. M. Repacholi, "Linking actions and emotions: evidence from 15- and 18-month-old infants," British Journal of Developmental Psychology, vol. 27, no. 3, pp. 649-667, 2009.

[8] N. Zmyj, D. Buttelmann, M. Carpenter, and M. M. Daum, "The reliability of a model influences 14-month-olds' imitation," Journal of Experimental Child Psychology, vol. 106, no. 4, pp. 208-220, 2010.

[9] D. Buttelmann, N. Zmyj, M. Daum, and M. Carpenter, "Selective imitation of in-group over out-group members in 14month-old infants," Child Development, vol. 84, no. 2, pp. 422428, 2013.

[10] G. Gergely, H. Bekkering, and I. Király, "Rational imitation in preverbal infants," Nature, vol. 415, p. 755, 2002.

[11] A. N. Meltzoff, "Infant imitation after a 1-week delay: long-term memory for novel acts and multiple stimuli," Developmental Psychology, vol. 24, no. 4, pp. 470-476, 1988.

[12] G. Gergely and G. Csibra, "Teleological reasoning in infancy: the naïve theory of rational action," Trends in Cognitive Sciences, vol. 7, no. 7, pp. 287-292, 2003.

[13] P. Rochat, "Object manipulation and exploration in 2- to 5month-old infants," Developmental Psychology, vol. 25, no. 6, pp. 871-884, 1989.

[14] D. Buttelmann, M. Carpenter, J. Call, and M. Tomasello, "Rational tool use and tool choice in human infants and great apes," Child Development, vol. 79, no. 3, pp. 609-626, 2008.

[15] A. Whiten, V. Horner, C. A. Litchfield, and S. Marshall-Pescini, "How do apes ape?" Learning and Behavior, vol. 32, no. 1, pp. 36-52, 2004.

[16] M. Tomasello, "Do apes ape?" in Social Learning in Animals: The Roots of Culture, C. M. Heyes and B. G. Galef Jr., Eds., pp. 319346, Academic Press, San Diego, Calif, USA, 1996.

[17] D. Buttelmann, M. Carpenter, J. Call, and M. Tomasello, "Enculturated chimpanzees imitate rationally," Developmental Science, vol. 10, no. 4, pp. F31-F38, 2007.

[18] M. Paulus, S. Hunnius, M. Vissers, and H. Bekkering, "Bridging the gap between the other and me: the functional role of motor resonance and action effects in infants' imitation," Developmental Science, vol. 14, no. 4, pp. 901-910, 2011.

[19] N. Zmyj and D. Buttelmann, "An integrative model of rational imitation in infancy," Infant Behavior and Development, vol. 37, no. 1, pp. 21-28, 2013.

[20] M. Beisert, N. Zmyj, R. Liepelt, F. Jung, W. Prinz, and M. M. Daum, "Rethinking 'rational imitation' in 14-month-old infants: a perceptual distraction approach," PLoS ONE, vol. 7, no. 3, Article ID e32563, 2012.

[21] A. Diamond and P. S. Goldman-Rakic, "Comparison of human infants and rhesus monkeys on Piaget's $A B$ task: evidence for 
dependence on dorsolateral prefrontal cortex," Experimental Brain Research, vol. 74, no. 1, pp. 24-40, 1989.

[22] K. E. Adolph, L. B. Karasik, and C. S. Tamis-LeMonda, "Using social information to guide action: infants' locomotion over slippery slopes," Neural Networks, vol. 23, no. 8-9, pp. 1033-1042, 2010.

[23] J. M. Mandler and L. McDonough, "Studies in inductive inference in infancy," Cognitive Psychology, vol. 37, no. 1, pp. 60-96, 1998.

[24] D. Buttelmann, M. Carpenter, J. Call, and M. Tomasello, "Chimpanzees, Pan troglodytes, recognize successful actions, but fail to imitate them," Animal Behaviour, vol. 86, no. 4, pp. 755-761, 2013.

[25] D. E. Lyons, A. G. Young, and F. C. Keil, "The hidden structure of overimitation," Proceedings of the National Academy of Sciences of the United States of America, vol. 104, no. 50, pp. 19751-19756, 2007.

[26] H. Over and M. Carpenter, "Putting the social into social learning: explaining both selectivity and fidelity in children's copying behavior," Journal of Comparative Psychology, vol. 126, no. 2, pp. 182-192, 2012.

[27] L. M. Hopper, A. Spiteri, S. P. Lambeth, S. J. Schapiro, V. Horner, and A. Whiten, "Experimental studies of traditions and underlying transmission processes in chimpanzees," Animal Behaviour, vol. 73, no. 6, pp. 1021-1032, 2007.

[28] M. Paulus, S. Hunnius, M. Vissers, and H. Bekkering, "Imitation in infancy: rational or motor resonance?" Child Development, vol. 82, no. 4, pp. 1047-1057, 2011.

[29] D. Buttelmann and N. Zmyj, "Evaluating the empirical evidence for the two-stage-model of infant imitation. A commentary on Paulus, Hunnius, Vissers, and Bekkering (2011)," Frontiers in Psychology, vol. 3, article 512, 2012.

[30] B. Elsner, "Infants' imitation of goal-directed actions: the role of movements and action effects," Acta Psychologica, vol. 124, no. 1, pp. 44-59, 2007.

[31] B. Eisner and B. Hommel, "Contiguity and contingency in action-effect learning," Psychological Research, vol. 68, no. 2-3, pp. 138-154, 2004.

[32] D. J. Lewkowicz, "Sensory dominance in infants: I. Six-monthold infants' response to auditory-visual compounds," Developmental Psychology, vol. 24, no. 2, pp. 155-171, 1988.

[33] D. J. Lewkowicz, "Sensory dominance in infants: II. Ten-monthold infants' response to auditory-visual compounds," Developmental Psychology, vol. 24, no. 2, pp. 172-182, 1988.

[34] C. W. Robinson and V. M. Sloutsky, "Auditory dominance and its change in the course of development," Child Development, vol. 75, no. 5, pp. 1387-1401, 2004.

[35] K. S. Bourgeois, A. W. Khawar, S. A. Neal, and J. J. Lockman, "Infant manual exploration of objects, surfaces, and their interrelations," Infancy, vol. 8, no. 3, pp. 233-252, 2005.

[36] R. M. Malina, "Motor development during infancy and early childhood: overview and suggested directions for research," International Journal of Sport and Health Science, vol. 2, pp. 5066, 2004. 


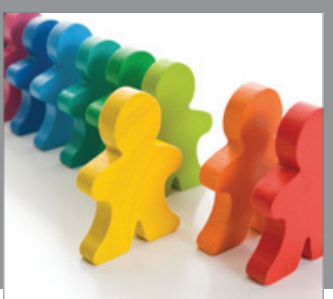

Autism

Research and Treatment
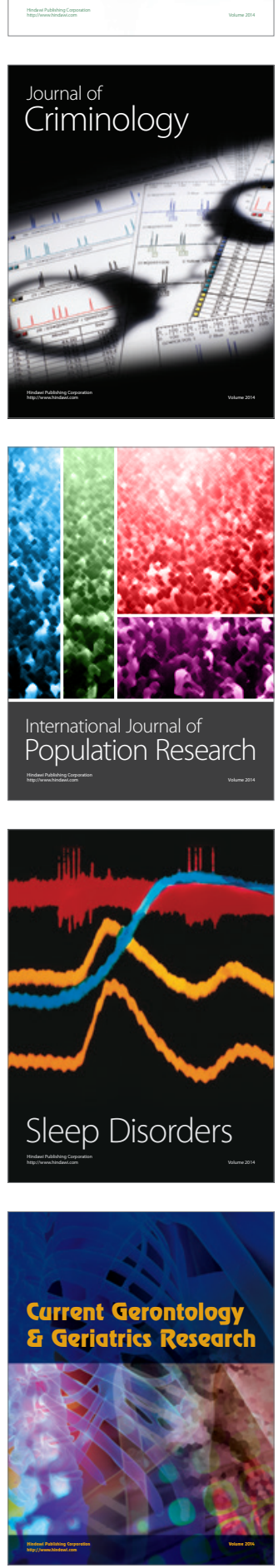

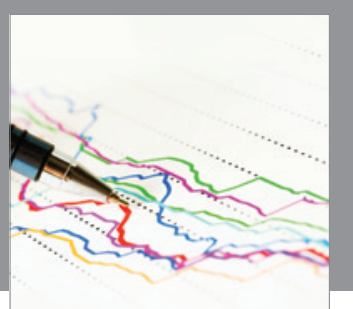

Economics

Research International
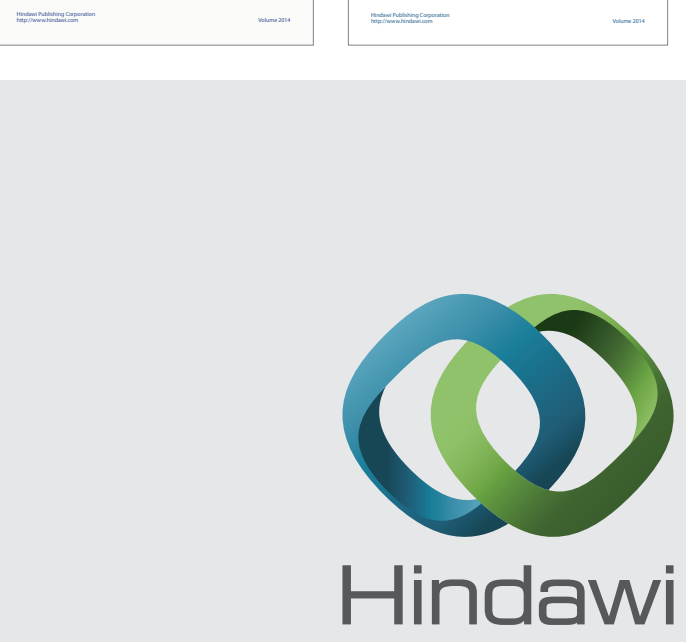

Submit your manuscripts at

https://www.hindawi.com
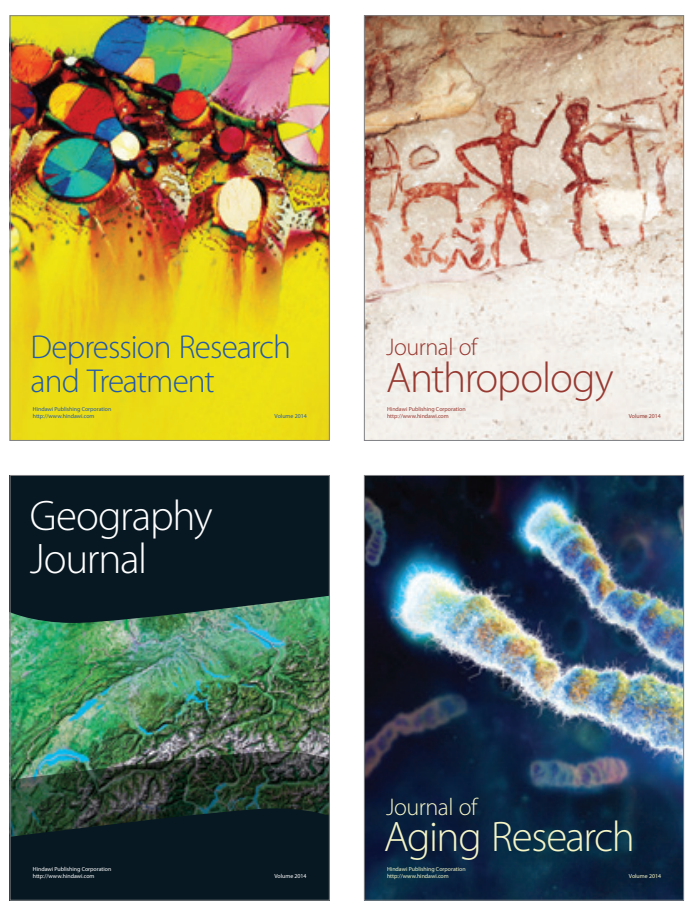
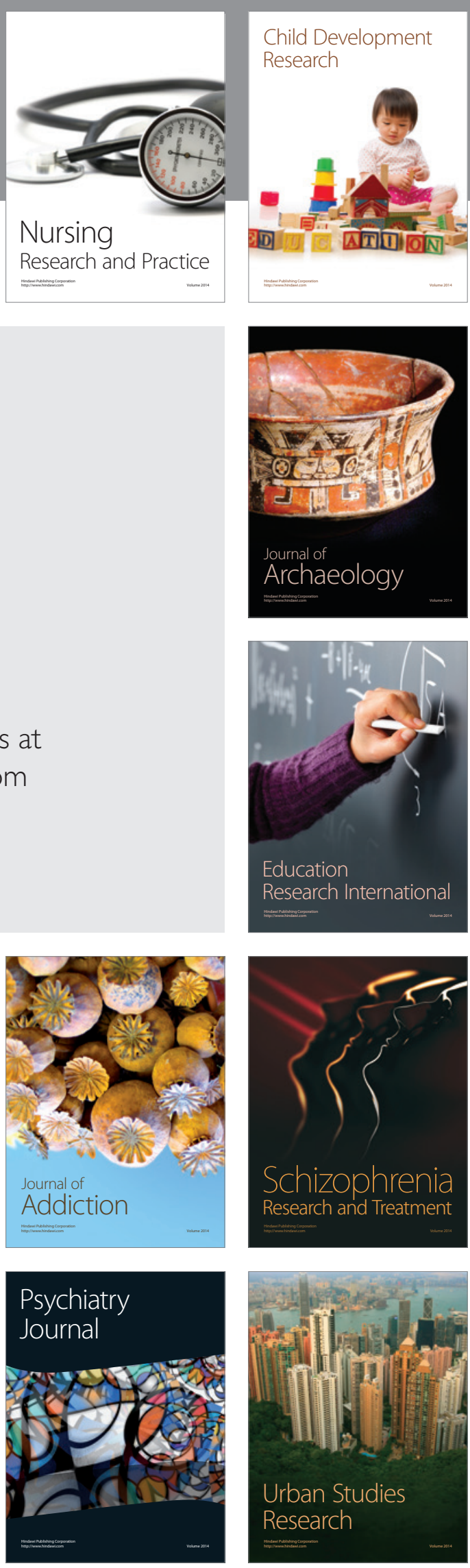